$65^{\text {th }}$ International Astronautical Congress, Toronto, Canada. Copyright $@ 2014$ by the International Astronautical Federation. All rights reserved.

IAC-14-A3.5.2

\title{
VENUS HIGH TEMPERATURE ATMOSPHERIC DROPSONDE AND EXTREME-ENVIRONMENT SEISMOMETER (HADES)
}

\author{
Nathan J. Boll \\ University of Michigan Ann Arbor, United States, njboll@umich.edu \\ Denise Salazar \\ University of Texas Austin, United States, denise.salazar.1210@gmail.com \\ Christopher J. Stelter \\ NASA Langley Research Center, United States, christopherstelter@gmail.com \\ Geoffrey A. Landis \\ NASA John H. Glenn Research Center, United States, geoffrey.landis@ nasa.gov \\ Anthony J. Colozza \\ NASA Glenn Research Center, United States, anthony.j.colozza@ nasa.gov
}

The atmospheric composition and geologic structure of Venus have been identified by the US National Research Council's Decadal Survey for Planetary Science as priority targets for scientific exploration, however the high temperature and pressure at the surface, along with the highly corrosive chemistry of the Venus atmosphere, present significant obstacles to spacecraft design that have severely limited past and proposed landed missions. Following the methodology of the NASA Innovative Advanced Concepts (NIAC) proposal regime and the Collaborative Modeling and Parametric Assessment of Space Systems (COMPASS) design protocol, this paper presents a conceptual study and initial feasibility analysis for a Discovery-class Venus lander capable of an extended-duration mission at ambient temperature and pressure, incorporating emerging technologies within the field of high temperature electronics in combination with novel configurations of proven, high Technology Readiness Level (TRL) systems. Radioisotope Thermal Power (RTG) systems and silicon carbide (SiC) communications and data handling are examined in detail, and various high-temperature instruments are proposed, including a seismometer and an advanced photodiode imager. The study combines this technological analysis with proposals for a descent instrument package and a relay orbiter to demonstrate the viability of an integrated atmospheric and in-situ geologic exploratory mission that differs from previous proposals by greatly reducing the mass, power requirements, and cost, while achieving important scientific goals.

\section{INTRODUCTION}

The HADES mission design study is the integrated effort of a multidisciplinary team of Research Associates from the NASA Space Academy at the John H. Glenn Research Center (GRC) under the direction of scientists and engineers from NASA Innovative Advanced Concepts (NIAC) and the Collaborative Modeling for Parametric Assessment of Space Systems (COMPASS) laboratory. The mission objectives are based on the priority science goals identified by the National Academy of Sciences' Planetary Science Decadal Survey, and are designed to explore the interior geologic structure and the atmospheric composition of Venus.

At its top level, this study is composed of three primary systems: a high temperature lander for in-situ seismology, an insulated descent package for comprehensive atmospheric observation, and a relay orbiter for communications and data handling. While each of these systems is considered in the analysis, the foremost goal of this paper is to investigate the subsystems of the lander, which present the most difficult challenges to conventional spacecraft design.

In addressing these challenges, HADES draws heavily from the heritage of previous Venus exploration missions, with the overall goal of significantly increasing the duration of operational deployment on the surface. Due to the harsh environment, where temperatures are consistently measured around 450 degrees Celsius $\left({ }^{\circ} \mathrm{C}\right)$ at over 92-bar atmospheric pressure, previous landers have survived for only brief periods. For example, the Soviet era spacecraft, Venera 13, was the longest-lived probe among such missions and was only capable of transmitting data for 127 minutes. ${ }^{2}$ In contrast, the HADES lander is designed to provide an operational window of one Venus solar day, the equivalent of approximately 117 Earth days, which would meet the requirements of all science objectives 
and represent a giant leap forward in technological progress.

Other recent proposals for Venus landers have attempted to meet similar mission lifetime targets. These designs plan to mitigate the extreme environmental challenges by providing passive or refrigerated pressure vessels within which lowtemperature equipment can be maintained. ${ }^{3,4}$ The high mass and extraordinary power requirements of such designs make them prohibitively expensive, however, ranging into the Flagship Program class. In contrast, the HADES lander utilizes recent advances in high temperature electronics and materials in order to operate at ambient temperature, thereby eliminating the need for expensive cooling systems and reducing the overall mass and mission cost.

Analysis of the lander incorporates a mission-wide scope, but focuses on key subsystems for detailed investigation and trade studies. The proposed science instrument package, which drives many of the requirements for other subsystems, describes the synthesis of current NASA capabilities with emerging technologies for the development of a high temperature seismometer, a photodiode imager, and a suite of atmospheric sensors. The power subsystem analysis trades the use of a customized radioisotope thermal generator with traditional battery power. Subsequently, further trade studies are performed for the communications (COM) and the command and data handling $(\mathrm{C} \& \mathrm{DH})$ subsystems, detailing the use of silicon carbide electronics in novel configurations.

\section{BACKGROUND AND ASSUMPTIONS II.I Venus Structure}

The physical structure of Venus is depicted in Fig. 1. Due to the historic absence of water, the atmosphere has evolved to be extremely dense and corrosive, composed of approximately $96.5 \%$ carbon dioxide (CO2) and $3.5 \%$ nitrogen (N2), with bands of thick sulfuric acid clouds between 30 and $70 \mathrm{~km}$ altitude. ${ }^{5,6}$ At the top of this cloud layer, where the temperature averages around $-43^{\circ} \mathrm{C}$, the winds are in a super-rotation and can reach speeds of up to $100 \mathrm{~m} / \mathrm{s}^{7}$ Beneath the cloud layer, the temperature and pressure are greatly increased, reaching $450^{\circ} \mathrm{C}$ and 92 bar at the surface, where the wind speed drops to approximately $0.5 \mathrm{~m} / \mathrm{s}^{\text {. }}$

Previous missions have remotely mapped the geomorphic features on the surface of Venus. This imagery has revealed randomly scattered craters over relatively recent volcanic activity, suggesting a surface age of no more than 500 million years. ${ }^{6}$ Beatty, et. al. (1999) stated that there is evidence of only "the last $10 \%$ of Venus's geological history recorded in its surface," thus very little is known about the internal structure and geologic activity of the planet, though it is assumed to possess a metallic core, rocky mantle, and thin crust similar to that of Earth. ${ }^{8}$

\section{II.II Previous Venus Missions and Proposals}

Due to the harsh nature of the Venus environment, only a small number of probes have successfully landed on its surface and transmitted data. These landers survived less than an hour on average, with the longestlived mission, Venera 13, lasting just 127 minutes. ${ }^{2}$ The most successful designs incorporated conventional (room temperature) electronics, insulated with phase change materials to resist the temperature and pressure at the surface. Recently proposed landers follow a similar concept, relying on refrigeration to maintain a cooled enclosure to house conventional instruments.

Because of the high power required to run a cooling system to reject heat from an actively-cooled enclosure to keep electronics at Earth-nominal temperatures in the high-temperature, high-pressure Venus environment, Venus lander design concepts that use an active refrigerator system are inherently large size and high power missions, and require development to bring the high-power dynamic power system and the cooler to technology readiness level suitable for flight. Assuming that the required technology is developed, such a large, high-power probe would be suitable for a "Flagship" class mission, but would be unlikely to be flown as a lower-cost mission, and hence is unlikely to be chosen for flight in a cost-restricted environment. It is the purpose of this project to design a mission which can be achieved at a lower cost by minimizing the power required, eliminating the cooled enclosure, and simplifying the mission design to focus on a small set of high-priority science goals. To do this, it was necessary to design the surface elements to use only components capable of operating in the high-temperature Venus environment, despite the relatively low performance capability of high-temperature electronics compared to the sophisticated microelectronic components available

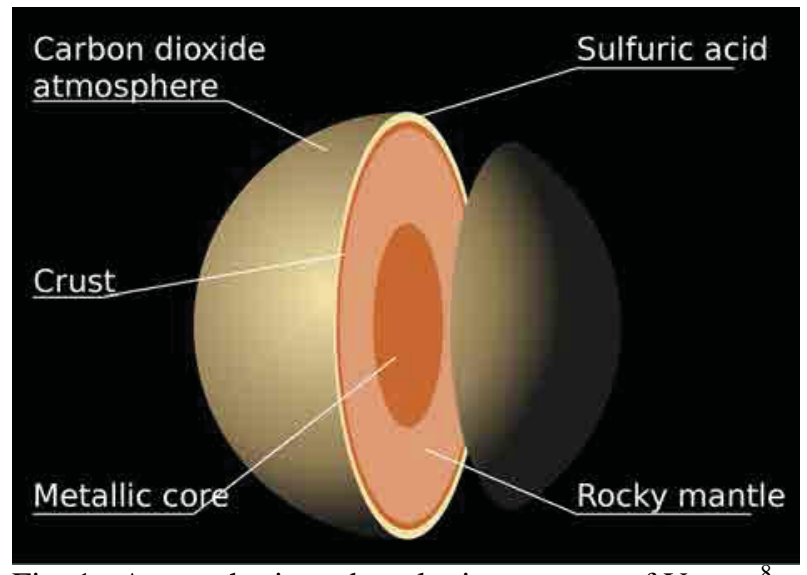

Fig. 1: Atmospheric and geologic structure of Venus. 
for operation at Earth temperatures. By keeping the mission simple and focused, we are able to baseline the use of a radioisotope thermoelectric generator (RTG) power source, which has no moving parts and is well demonstrated on previous missions (although not previously at Venus temperatures).

The primary science for the landed mission was chosen to be a seismometer package. This is one of the highest priority science objectives for Venus, since at the moment there is no knowledge of the interior structure of Venus. A detailed seismic study of Venus would require multiple seismometers at different locations; this could be done with several copies of the design envisioned here, although the present study was limited to a single probe design, and did not examine the requirements of multiple landed stations.

In addition to the landed science, the HADES probe also takes scientific measurements during the descent, to get a profile of the atmosphere. Since the descent duration is short compared to the long-duration surface mission, this package can be kept at terrestrial operating conditions for the limited time that the descent is in the hot lower atmosphere, and hence can use more sophisticated electronics.

Portions of the HADES analysis rely heavily on heritage from previous missions, including the Advanced Lithium Ion Venus Explorer (ALIVE) and the Venus Intrepid Tessera Lander (VITaL) proposals. ${ }^{4,9}$ Together this heritage informs many of the science objectives, design choices, and the concept of operations (CONOPS) of this study.

\section{II.III Assumptions and Approach}

The launch vehicle, cruise stage, and basic design of entry, descent and landing (EDL) are assumed from heritage, and no detailed analyses of these mission components were performed. The study remains focused on the three primary instrument platforms to be deployed in-situ, including a lander, descent package, and orbiter, using the subsystem classifications:

- Science Subsystem

- Communications (COM)

- $\quad$ Command \& Data Handling (C\&DH)

- Guidance, Navigation \& Control (GN\&C)

- Power

- Propulsion

- $\quad$ Structures \& Mechanisms (SMS)

- Thermal Control

II.IV Requirements and Constraints II.IV.I Lander

- Science mode: 1 Venus solar day (117 Earth days)

- All subsystems operational at $450^{\circ} \mathrm{C}$

- Target landing site near the North Pole

- No data storage, uplink to orbiter only

\section{II.IV.II Descent Package}

- Heritage instruments, high TRL

- Phase change materials provide insulation

- Operational during entire descent

- High data rate communication with orbiter

\section{II.IV.III Orbiter}

- Heritage spacecraft design

- Direct to Earth communication

- Highly elliptical polar orbit

- Contact with lander: $75 \%$ of science mode

\section{II.V Margin Policy}

Mass and power growth is chosen to be $30 \%$ for the lander and $10 \%$ for the descent package. The descent package is primarily composed of off-the-shelf, high TRL technologies, while the lander has overall low TRL and is therefore given a larger margin.

\section{II.VI Mission Description}

The mission is composed of a lander, descent package, and orbiter transferred to polar orbit around Venus. The lander is delivered to the surface near the North Pole by means of an aeroshell where it deploys a seismometer for geologic observation, as well as an imager and meteorological sensors. The aeroshell also contains the insulated descent package of science instruments that perform as a dropsonde, taking comprehensive atmospheric readings upon entry. The orbiter remains in polar orbit to serve as the data relay for communication with Earth and may also contain remote sensing instruments.

\section{II.VI.I Mission Trades}

The two primary trade studies involved the communications and power subsystems, respectively. The communications trade study determines the orbital parameters for achieving optimal data collection from the lander by the orbiter. The trade study for the power subsystem compares the use of a customized radioisotope power system with primary batteries.

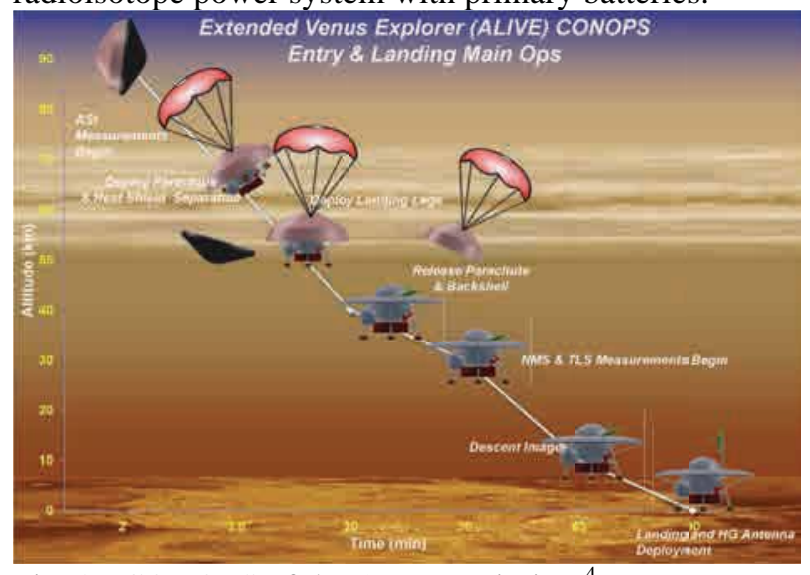

Fig. 2: CONOPS of the ALIVE mission. ${ }^{4}$ 


\section{II.VI.II CONOPS}

The concept of operations focuses on the mission once the spacecraft has already reached an orbit around Venus. Fig. 2 displays the mission CONOPS for the ALIVE mission proposal. HADES will follow a similar series of operations, with precise altitudes for entry, drogue chute deployment, and separation of the lander from the descent package to be calculated in future study. The following is a chronological list of each step of the mission starting from orbit insertion.

- The spacecraft will enter a highly elliptical polar orbit around Venus.

- The aeroshell will separate from the orbiter.

- The aeroshell will enter the atmosphere of Venus at approximately $90 \mathrm{~km}$ altitude.

- A drogue parachute will be deployed to stabilize the aeroshell and decrease velocity.

- The heat shield will separate from the bottom of the aeroshell, exposing the lander.

- The lander will separate from the aeroshell.

- The descent package inside the aeroshell is expected to continue to drift for approximately 5 hours under parachute.

- The lander will impact the surface and remain in place, operating in science mode for 117 days.

\section{II.VI.III Mission Communication Details}

The orbiter will achieve a highly elliptical polar orbit around Venus. It will spend most of its time above the North Pole where the lander will be deployed. The lander will uplink data continuously to the orbiter without any downlink commands from the orbiter. When the lander is out of the field of view of the orbiter, the lander will continue to send data but the orbiter will not be listening. It is estimated that the orbiter will capture $75 \%$ of the data that the lander transmits. The orbiter will relay all data using direct to Earth (DTE) communications.

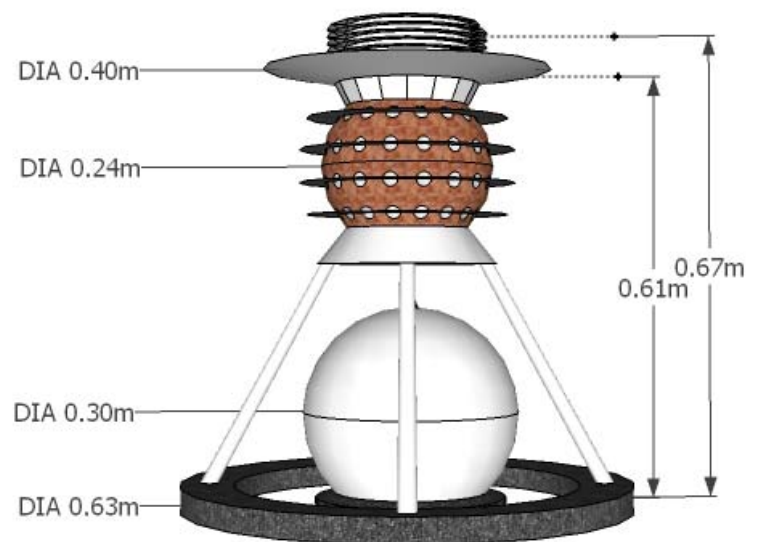

Fig. 3: HADES lander conceptual design showing the estimated dimensions in science mode.

\section{BASELINE DESIGN}

\section{III.I Top-Level Design}

Figs. 3 and 4 display the conceptual design for the lander. A zenith pointing helical antenna and the COM and C\&DH subsystems are located on top of a spherical radioisotope power system (RPS). The antenna has a diameter of 0.4 meters and the RPS has a diameter of 0.24 meters. The science package, including the high temperature seismometer (HTS), atmospheric structure instrument (ASI), gas sensor array (GSA), and the high temperature imager (HTI), is below the RPS. Four struts and an impact-absorbing ring support the platform. Total deployed height of the lander is 0.67 meters.

III.I.I Mass Equipment List (MEL)

The MEL for the lander and descent package are displayed in tables 1 and 2. The communications subsystem masses for both the lander and descent package are estimates. The mass of the command and data handling subsystem of the lander is assumed to be $67 \%$ of the science package mass, initially estimated at 5 kilograms. The final masses of the lander and subsystem are therefore 96 and 102 kilograms respectively. The total masses of the other subsystems will be detailed throughout the paper.

\begin{tabular}{lrrrr}
\hline & $\begin{array}{r}\text { Mass } \\
(\mathrm{kg})\end{array}$ & $\begin{array}{r}\text { Growth } \\
(\%)\end{array}$ & $\begin{array}{r}\text { Growth } \\
(\mathrm{kg})\end{array}$ & $\begin{array}{r}\text { Total } \\
(\mathrm{kg})\end{array}$ \\
\hline Science Pkg. & 15.5 & 30 & 4.7 & 20.2 \\
COM & 9 & 30 & 2.7 & 11.7 \\
C\&DH & 10 & 30 & 3 & 13 \\
GN\&C & 0 & 30 & 0 & 0 \\
Power & 24 & 30 & 7.2 & 31.2 \\
Propulsion & 0 & 30 & 0 & 0 \\
SMS & 15 & 30 & 4.5 & 19.5 \\
Thermal & 0 & 30 & 0 & 0 \\
Lander & $\mathbf{7 3 . 5}$ & $\mathbf{3 0}$ & $\mathbf{2 2 . 1}$ & $\mathbf{9 5 . 6}$ \\
\hline
\end{tabular}

Table 1: MEL for lander subsystems.

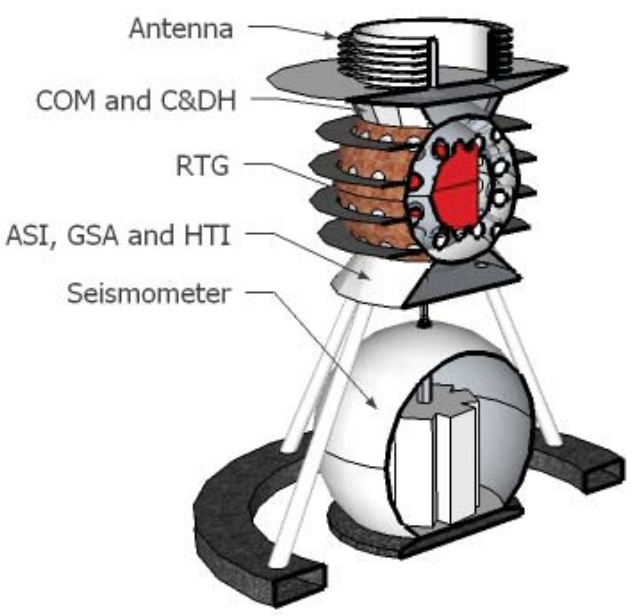

Fig. 4: HADES lander conceptual design cutaway. 


\begin{tabular}{lrrrr}
\hline & $\begin{array}{r}\text { Mass } \\
(\mathrm{kg})\end{array}$ & $\begin{array}{r}\text { Growth } \\
(\%)\end{array}$ & $\begin{array}{r}\text { Growth } \\
(\mathrm{kg})\end{array}$ & $\begin{array}{r}\text { Total } \\
(\mathrm{kg})\end{array}$ \\
\hline Science Pkg. & 22.3 & 10 & 2.2 & 24.5 \\
COM & 4 & 10 & 0.4 & 4.4 \\
C\&DH & 5 & 10 & 0.5 & 5.5 \\
GN\&C & 0 & 10 & 0 & 0 \\
Power & 9.2 & 10 & 0.9 & 10.2 \\
Propulsion & 0 & 10 & 0 & 0 \\
SMS & 2 & 10 & 0.2 & 2.2 \\
Thermal & 50 & 10 & 5 & 55 \\
Descent Pkg. & $\mathbf{9 2 . 5}$ & $\mathbf{1 0}$ & $\mathbf{9 . 2}$ & $\mathbf{1 0 1 . 8}$ \\
\hline
\end{tabular}

Table 2: MEL for descent package subsystems.

\section{III.I.II Power Equipment List (PEL)}

The only power mode considered in this study is the science mode once the descent package has entered the atmosphere and the lander is on the surface of Venus. Tables 3 and 4 display the PEL for the lander and descent package in science mode. The power required for the communications subsystem of the lander is based on a link budget showing 0.1 Watts Radio Frequency (RF) power required. The power required for the command and data handling subsystem of the lander is based on a proof of concept circuit developed later in this paper. It includes an estimate of per-element power consumption. The power required for the communications subsystem of the descent package based on a rough estimate of a link budget. The power required for the command and data handling subsystem

\begin{tabular}{lrrrr}
\hline & $\begin{array}{r}\text { Power } \\
(\mathrm{W})\end{array}$ & $\begin{array}{r}\text { Growth } \\
(\%)\end{array}$ & $\begin{array}{r}\text { Growth } \\
(\mathrm{W})\end{array}$ & $\begin{array}{r}\text { Total } \\
(\mathrm{W})\end{array}$ \\
\hline Science Pkg. & 8.2 & 30 & 2.5 & 10.7 \\
COM & 3.3 & 30 & 1 & 4.3 \\
C\&DH & 1.1 & 30 & 0.33 & 1.4 \\
GN\&C & 0 & 30 & 0 & 0 \\
Power & 0 & 30 & 0 & 0 \\
Propulsion & 0 & 30 & 0 & 0 \\
SMS & 0 & 30 & 0 & 0 \\
Thermal & 0 & 30 & 0 & 0 \\
Lander & $\mathbf{1 2 . 6}$ & $\mathbf{3 0}$ & $\mathbf{3 . 8 3}$ & $\mathbf{1 6 . 4}$ \\
\hline
\end{tabular}

Table 3: PEL for lander subsystems.

\begin{tabular}{lrrrr}
\hline & $\begin{array}{r}\text { Power } \\
(\mathrm{W})\end{array}$ & $\begin{array}{r}\text { Growth } \\
(\%)\end{array}$ & $\begin{array}{r}\text { Growth } \\
(\mathrm{W})\end{array}$ & $\begin{array}{r}\text { Total } \\
(\mathrm{W})\end{array}$ \\
\hline Science Pkg. & 79.7 & 10 & 7.97 & 87.7 \\
COM & 25 & 10 & 2.5 & 27.5 \\
C\&DH & 10 & 10 & 1 & 11 \\
GN\&C & 0 & 10 & 0 & 0 \\
Power & 0 & 10 & 0 & 0 \\
Propulsion & 0 & 10 & 0 & 0 \\
SMS & 0 & 10 & 0 & 0 \\
Thermal & 0 & 10 & 0 & 0 \\
Descent Pkg. & $\mathbf{1 1 4 . 7}$ & $\mathbf{1 0}$ & $\mathbf{1 1 . 4 7}$ & $\mathbf{1 2 6 . 2}$ \\
\hline
\end{tabular}

Table 4: PEL for descent package subsystems. of the descent package is based on a powerful computer package that is ten times that of a radiation-hardened, Version 8 Scalable Processor ARChitecture (SPARC V8) processor. The power requirements for the other subsystems will be described throughout the paper. Total power requirement for the lander and descent package are 16 Watts and 126 Watts respectively.

\section{SUBSYSTEM BREAKDOWN}

\section{IV.I Science Package}

The HADES science package consists of two separate systems, an insulated descent package and a suite of high temperature surface instruments. The descent package draws exclusively from high TRL heritage technology, thus only a brief summary is included for each element, while a more detailed analysis of the surface instruments is provided.

\section{IV.I.I Descent Science Instruments}

The descent science instrument package described in Table 5 incorporates atmospheric sensors and a high definition imager, permanently housed in the backshell portion of the aeroshell. It is assumed to be independently powered by lithium ion (Li-ion) batteries, thermally controlled using phase change salts to operate at low temperature during the final $90 \mathrm{~km}$ of EDL, and capable of high rates of data transmission. Because this package is not required to operate at the high temperatures of the Venus surface, much of the required technology will be directly adapted from heritage missions. The measurements collected by this configuration of instruments will directly address a number of the highest priority questions identified by the 2013 Decadal Survey concerning the physics, chemistry, geology, and mechanical dynamics driving the atmosphere and climate on Venus.

Descent Atmospheric Structure Instrument (DASI): DASI is a suite of meteorological instruments that incorporates several atmospheric sensors for measuring temperature, pressure, and wind velocity, as well as an accelerometer to determine density and descent motion. This configuration would be similar to previous descent packages, such as Mars Pathfinder, calibrated for Venus conditions during operation, and would provide detailed profiles of the Venusian atmosphere across various altitudes. ${ }^{10}$

Ion and Neutral Mass Spectrometer (INMS): Adapted from the Cassini mission, the INMS is a quadrupole mass spectrometer designed to determine the composition and structure of ions and neutral particles in the Venus atmosphere by varying the applied voltage across two pairs of metal rods. ${ }^{11}$

Tunable Laser Spectrometer (TLS): TLS uses a diode laser and second-harmonic detection for in situ trace-gas measurement. This instrument is capable of 
detecting a variety of important trace elements in the Venus atmosphere, including organic compounds, and will be adapted from the Mars Science Laboratory (MSL) mission. ${ }^{12}$

Net Flux Radiometer (NFR): Drawing on heritage from the Galileo and Pioneer Venus missions, the NFR instrument will use a high temperature flux plate to investigate the stratification and distribution of radiative energy dynamics, which drive Venusian atmospheric circulation. $^{13,14}$

Magnetometer: The magnetosphere of Venus is substantially different from that of Earth and is of high interest for further investigation. A flux magnetometer, similar to previous instruments flown on Pioneer Venus and Cassini, will record these measurements at various altitudes. $^{15,16}$

Nephelometer: The nephelometer instrument, as previously flown on Pioneer Venus and Galileo, will measure the components of the aerosol phase matrix of the Venus atmosphere, characterizing the vertical extent, structure, and microphysical attributes of the cloud layers. ${ }^{17}$

Lightning Detector: Evidence for lightning on Venus has been observed previously, though it has been suggested that the most likely form is intra-cloud lightning in the upper atmosphere. ${ }^{18} \mathrm{~A}$ modified lightning detector will observe the characteristic radio signatures associated with lightning activity. ${ }^{19}$

Venus Descent Imager (VENDI): At altitudes below $15 \mathrm{~km}$ above the surface, the Venus atmosphere will be clear enough for the surface to be imaged. ${ }^{7}$ By employing a high-definition video instrument, similar to the Mars Descent Imager (MARDI) flown on MSL, VENDI will provide the highest quality images of the surface of Venus ever captured. ${ }^{20}$ In addition to their inherent exploratory value, these images will provide geologic and geomorphic context for the landing sight and surface science package.

\begin{tabular}{lrrrr}
\hline & $\begin{array}{r}\text { Mass } \\
(\mathrm{kg})\end{array}$ & $\begin{array}{r}\text { Power } \\
(\mathrm{W})\end{array}$ & $\begin{array}{r}\text { Data } \\
(\mathrm{kbps})\end{array}$ & $\begin{array}{r}\text { Volume } \\
\left(10^{3} \mathrm{~cm}^{3}\right)\end{array}$ \\
\hline DASI & 2.1 & 3.5 & 0.3 & 1.0 \\
INMS & 12 & 55 & 0.6 & 16 \\
TLS & 5.0 & 18 & 1.1 & 2.5 \\
NFR & 2.5 & 5.1 & .28 & 1.5 \\
Magnetometer & 1.1 & 2.2 & 0.3 & 3.0 \\
Nephelometer & 0.6 & 1.3 & 0.3 & 1.5 \\
Lightning Det. & 0.6 & 0.6 & 0.3 & 1.0 \\
VENDI & 0.6 & 1.3 & 1.8 & 2.3 \\
Descent Pkg. & $\mathbf{2 4 . 5}$ & $\mathbf{8 7}$ & $\mathbf{4 . 9 8}$ & $\mathbf{2 8 . 8}$ \\
\hline
\end{tabular}

Table 5: HADES candidate descent instrument payload.

\section{IV.I.II Surface Science Instruments}

The surface science instrument package described in Table 6 includes the High Temperature Seismometer (HTS) and High Temperature Imager (HTI), along with two sensor suites, the Surface Atmospheric Structure
Instrument (SASI) and the Gas Sensor Array (GSA), calibrated for meteorological, structural and chemical observations of the atmosphere. This package is notionally developed from the heritage of previous missions in conjunction with extreme environment solutions adapted from various scientific disciplines and emerging technologies currently under development at GRC. As such, the proposed designs each demonstrate low TRL and only the mass and power requirements were analyzed. Significant development and testing, using the Glenn Extreme Environment Rig (GEER), will be required to advance the readiness of these instruments and determine the data transmission requirements, in detail. ${ }^{21}$ Thus, for the purposes of this study, it is assumed that this notional package will be constructed to conform to the limits of the proposed communications system, currently estimated at 2-4 kbps at an operational temperature of $450^{\circ} \mathrm{C}$.

High Temperature Seismometer (HTS): The toplevel design for the HTS is based on the Seismic Experiment for Interior Structures (SEIS) instrument in Fig. 5, currently under development for the Interior Exploration using Seismic Investigations, Geodesy and Heat Transport (InSight) mission to Mars. This instrument is composed of three Very Broad Band (VBB) spring leaf geophones and three short band Microelectromechanical Systems (MEMS) seismic sensors, contained within a self-leveling pressure vessel to isolate the sensor heads from the atmosphere and to reduce temperature variations. ${ }^{22}$ The Hades mission requires the modification of each component to ensure operation in the Venus environment.

VBB capacitive geophones have recently been demonstrated at GRC to operate at $500^{\circ} \mathrm{C}$ with a predicted sensitivity from $1-30 \mathrm{~Hz}^{23}$ This will provide the necessary resolution to observe long-wavelength seismic activity with a simple, analog sensor.

Commercially available, high temperature, shortband MEMS accelerometers currently operate at $175^{\circ} \mathrm{C} .{ }^{24}$ While recent advances in silicon carbide (SiC) electronics will provide increased performance, further study is required to meet the mission objectives. As this
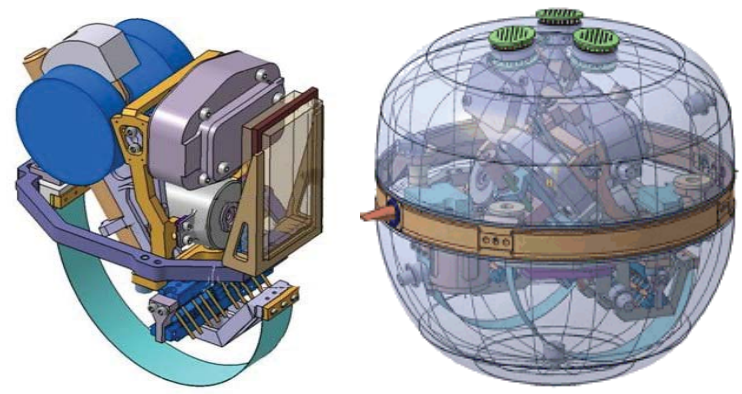

Fig. 5: VBB spring leaf geophone (left) and a notional 3 -axis configuration inside a pressure vessel (right) for the SEIS seismic instrument. ${ }^{25}$ 
technology continues to improve, however, it may become possible to employ digital MEMS sensors, exclusively.

To address the physical demands of the Venus environment, HADES draws from terrestrial seismic research. Deep-sea seismometer housings currently withstand pressures significantly greater than the atmospheric pressure on Venus and could be constructed of the appropriate materials to resist corrosion and allow for thermal expansion. ${ }^{26}$ Further study will be required to develop an efficient coupling mechanism to ensure low-noise in-situ observation.

The HTS instrument will be physically decoupled from the lander upon touchdown with the use of shape memory alloys, as described in the Structures and Mechanisms subsystem.

High Temperature Imager (HTI): The HTI instrument is primarily composed of high temperature photodiodes and a scanning mirror designed to capture high definition panoramic images of the surface of Venus throughout the duration of the mission. The preliminary design of HTI is based on the cameras flown on the Viking Mars lander, which used 12 silicon photodiodes to capture high definition images in grayscale, color and the near infrared with an angular resolution of $0.12^{\circ}$ between $-60^{\circ}$ and $+40^{\circ}$ elevation. $^{27}$ The components of these cameras would be modified with recently developed technologies to achieve a significantly improved performance in the Venus environment.

New photovoltaic materials, such as gallium indium phosphide, have been developed and tested at GRC, and are shown to function in triple junction photovoltaic cells at temperatures of up to $400^{\circ} \mathrm{C}$. This will allow for an increase in the density of photodiodes within the sensor head of the instrument, leading to higher resolution imaging over a broader spectral range. ${ }^{28}$

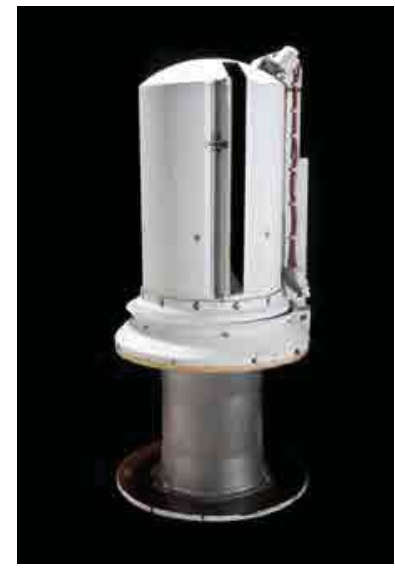

Fig. 6: A back-up camera identical to the original photodiode camera of the Viking Lander mission to mars: $40.64 \mathrm{~cm}$ tall $\mathrm{x} 21.59 \mathrm{~cm}$ wide, $7.3 \mathrm{~kg}$. ${ }^{29}$



Fig. 7: GSA configuration. ${ }^{30}$

Although the camera design is simple, high temperature motors will be required to control the scanning mirror on two axes. Honeybee Robotics has demonstrated miniaturized Switched Reluctance Motors (SRM) operating at $460^{\circ} \mathrm{C}$, which will be employed to actuate the azimuth rotation and elevation scan axes and which will allow for reductions in size and mass. ${ }^{31}$

The HTI will be statically housed behind a sapphire window, with only the scanning mirror in motion. The power requirements of the HTI currently limit the system to a single camera. This configuration cannot provide $360^{\circ}$ coverage around the lander, and further study should be conducted in order to reduce power demands and add an additional camera.

Gas Sensor Array (GSA): The GSA shown in Fig. 7 is a chemical sensor suite constructed using MEMS and SiC-based semiconductor technology. These sensors were originally developed for chemical species detection in hazardous environments, such as rocket and jet engines, with support from the NASA Small Business Innovation Research (SBIR) and Small Business Technology Transfer (STTR) programs. ${ }^{32}$ The sensor suite will mount directly to the structure of the lander and will require no moving parts.

Surface Atmospheric Structure Instrument (SASI): The SASI sensor suite will analyze the physical properties and meteorological conditions of the Venus atmosphere, such as temperature, pressure, and wind speed. These results will be correlated with data from the other instruments to provide context and enhance performance, such as improving the signal to noise ratio in the seismometer, and to better understand the dynamics of the Venus environment. Similar instrument configurations have been previously deployed on the Venera, Huygens and Mars Pathfinder missions. ${ }^{33,10}$

\begin{tabular}{lrrr}
\hline & $\begin{array}{r}\text { Mass } \\
(\mathrm{kg})\end{array}$ & $\begin{array}{r}\text { Power } \\
(\mathrm{W})\end{array}$ & $\begin{array}{r}\text { Volume } \\
\left(10^{3} \mathrm{~cm}^{3}\right)\end{array}$ \\
\hline HTS & 6.5 & 1.3 & 27 \\
HTI & 9.8 & 3.9 & 3.4 \\
GSA & 1.3 & 1.3 & 1.0 \\
SASI & 2.6 & 4.2 & 1.0 \\
Science Pkg. & $\mathbf{2 0 . 2}$ & $\mathbf{1 0 . 7}$ & $\mathbf{3 2 . 4}$ \\
\hline
\end{tabular}

Table 6: HADES candidate surface instruments. 
IV.II Communications Subsystem (COM)

IV.II.I COM Requirements and Assumptions

- Lander shall uplink data to orbiter continuously.

- There is no downlink or commanding of the lander. All lander operations are fully autonomous.

- Lander shall be positioned near the pole and the orbiter shall be in a near polar orbit.

- There will be $75 \%$ availability of data.

\section{IV.II.II Signal to Noise Calculation}

Equation (1) displays the Shannon-Hartley Channel Capacity Theorem:

$$
C=B \log _{2}\left(1+\frac{S}{N}\right)
$$

Where $C$ is the channel capacity (in bits per second), $B$ is the bandwidth (in Hertz), $S$ is the signal power (in Watts) and $N$ is the noise power. This is an expression for the greatest possible error-free bit rate given a certain bandwidth and signal-to-noise ratio. Modern efficient error correcting algorithms (such as turbo codes) can approach the theoretical channel capacity, but we will be limited to a fraction of this limit because of the necessarily crude electronics package of the lander. For the purpose of electronic and analytical simplicity, an extended Hamming code will be used for forward-error-correction (and detection) while a binary phase-shift key (BPSK) scheme will be used for modulation. Demodulation and error correcting on the relay satellite (or on the ground) is not thought to present any special constraints, so is considered out of the scope of this paper.

A Hamming code is a non-trivial (meaning, in our context, useful for proof-of-concept) error-correcting code, which was implemented via exclusive-or (XOR) gates. A Hamming code of length $2^{l}-1$ requires $l$ parity bits and encodes $2^{l}-1-l$ data bits allowing any single error to be corrected (for an integer $l>1$ ). The common Hamming $(7,4)$ code has a length of 7 bits, 4 of which are data bits (the remaining 3 bits are the parity bits). In our case, while correcting errors is useful, detecting errors is arguably more critical. We implemented a Hamming $(15,11)$ code with an extra parity bit to allow single-error-correction and dualerror-detection in a total length of 16 bits with 11 data bits. The data from our instruments is in a 10-bit format, and because of the nature of Hamming codes, it was convenient to add another bit to allow additional information to be sent simultaneously (for instance, the state of a counter). For a $\operatorname{Hamming}(m, n)$ code the probability $P_{r}$ of receiving the $n$ data bits correctly with a raw bit error probability of $q$ can be shown in Eq. (2):

$$
P_{r}=(1-q)^{m}+m q(1-q)^{m-1}
$$

In this example, the last extra parity bit is irrelevant to the calculation of probability of error-free, so our 16bit-length extended Hamming code has a probability of successful full-integrity data transmission of in Eq. (3):

$$
P_{r}=(1-q)^{15}+15 q(1-q)^{14}
$$

If two errors occur, the data cannot be recovered, but the double-error condition can be detected. In general, for an extended Hamming code (capable of single-error correction and double-error detection) with $n$ data bits and total length of $m$ bits, it can be shown that the probability of getting two or fewer errors (and thus being guaranteed of detecting the error) is (Eq. (4)):

$$
\begin{array}{rl}
P_{r}=(1-q)^{m}+m & q(1-q)^{m-1} \\
& +q^{2} \frac{m}{2}(m-1)(1-q)^{m-2}
\end{array}
$$

As mentioned earlier, we will tolerate a word nondetected error probability (and thus $1-P_{r}$ ) of $10^{-6}$. Solving the above equation, we get a maximum bit error probability of $q=0.001218$ (here and elsewhere, only the first non-zero digit is really significant). For a binary phase-shift key (BPSK) modulation scheme, it can be shown in Eq. (5) that the bit error probability is:

$$
q=\frac{1}{2} \operatorname{erfc}\left(\sqrt{\frac{s}{N}}\right)
$$

Where erfc is the complementary error function in Eq. (6):

$$
\operatorname{erfc}(x)=\frac{2}{\sqrt{\pi}} \int_{x}^{\infty} e^{-t^{2}} \mathrm{~d} t
$$

Solving for the signal-to-noise ratio $(S / N$ or $\mathrm{SNR})$, we get in Eq. (7):

$$
S / N=4.594
$$

The noise power $N$ can be found by Eq. (8):

$$
N=k_{b} B T
$$

Where $B$ is the bandwidth (in Hertz) over which the noise is integrated, $T$ is the system noise temperature in Kelvin, and $k_{b}$ is the Boltzmann constant. For a BPSK modulation scheme, the bandwidth of the signal is approximately the symbol rate, i.e. the raw bit rate (data bits plus parity bits). At this point, the analysis will be truncated in the interest of constraining the scope of this 
paper and a system noise temperature of 450 Kelvin will be chosen as a design constraint of the relay orbiter. The total required bitrate $C$ is given as the sum of each instrument's bitrate plus a $30 \%$ growth margin, giving a total information rate of 3000 bits per second and thus a channel bandwidth $B$ of $3000 \mathrm{~Hz}$. Finally we get a noise power of $N=1.864 \cdot 10^{-17}$ Watts and thus a signal power (at the receiver) requirement in Eq. (9) of:

$$
S=8.563 \cdot 10^{-17} \text { Watts }
$$

\section{IV.II.III Transmission}

Friis Transmission Equation is shown in Eq. (10):

$$
\frac{S}{P_{R F}}=G_{r} \cdot G_{t} \cdot\left(\frac{\lambda}{4 \pi l}\right)^{2} \eta_{\text {medium }}
$$

Where $\eta_{\text {medium }}$ is the efficiency of the medium, i.e. what is left after absorption losses, $\lambda$ is the wavelength, $l$ is the distance from transmitter to receiver, and $G_{r}$ and $G_{t}$ are the gains of the receiver and transmitter, respectively, and $P_{R F}$ is the power of the signal coming out of the RF amplifier on the lander. The squared term is the part traditionally referred to as free space loss. Gain for an antenna can be expressed as the as the directivity times an efficiency coefficient, where the directivity for a parabolic antenna is related to the aperture area and wavelength is found in Eq. $(11)^{34}$ :

$$
G_{r}=\eta_{\text {aper }, r} \frac{\pi^{2} d_{r}^{2}}{\lambda^{2}}
$$

Where $\eta_{\text {aper }}$ in this case is the aperture efficiency of the antenna (can range from .55 to .7), $d_{r}$ is the aperture diameter, and $\lambda$ is the wavelength. For the lander's antenna, it is assumed that the antenna will point straight up (i.e. in the opposite direction of the local gravity gradient), that the antenna will be stationary, and that the lander will remain directly below the relay satellite's apoapsis. In order to provide modest requirements to the relay orbit, it is assumed that the antenna will provide a $90^{\circ} 3 \mathrm{~dB}$ beamwidth in order to fit the $75 \%$ data availability requirement. So the beam width of the lander antenna actually drives the orbit of the relay satellite. The lander is at the surface of Venus (which has a radius of about 6050 kilometers), and it is assumed the relay satellite has a periapsis of no more than $400 \mathrm{~km}$ altitude (so a periapsis of about 6450 kilometers). We can figure out the apoapsis from Kepler's Second Law ("A line from the satellite to the central body sweeps over equal areas in equal intervals of time."). he swept area must be $75 \%$ of the area of the entire orbit ellipse. Solving the relevant equations using Mathematica such that the swept area was $75 \%$ of the total ellipse area, we get an apoapsis of about 61900 kilometers. Thus, the maximum distance $l$ between the lander and relay satellite is $l=61900$ kilometers 6050 kilometers $=55850$ kilometers. The lander antenna is designed in such a way that its beamwidth is $90^{\circ}$, and since we can express gain in Eq. (12):

$$
G_{t}=\eta_{\text {aper }} \frac{4 \pi}{\Delta \Omega}
$$

Where $\Delta \Omega$ is the solid angle of the beam (assuming all the beam energy is focused evenly inside the beam width) and $\eta_{\text {aper }}$ is the aperture efficiency (typically, $.55<\eta_{\text {aper }}<.7$ for a parabolic antenna with $\lambda \ll d$ ).

$\Delta \Omega$ can be found by integrating the area of a portion of the unit circle in Eq. (13):

$$
\begin{aligned}
\Delta \Omega & =\int_{0}^{2 \pi} \int_{0}^{\frac{\theta_{b}}{2}} \sin \theta \mathrm{d} \theta \mathrm{d} \phi \\
& =2 \pi\left(1-\cos \left(\theta_{b} / 2\right)\right)
\end{aligned}
$$

Which gives Eq. (14):

$$
G_{t}=\eta_{\text {aper }, t} \frac{2}{1-\cos \left(\theta_{b} / 2\right)}
$$

Where for $\theta_{b}=90^{\circ}$ and $\eta_{\text {aper }}=1$ (for simplifying the analysis), we get a gain of $G_{t} \sim 6.828$. The equation for the size of an antenna to achieve this gain can be approximated by the parabolic equation: $0.69 \lambda=d_{t}$, from which we can see that the diameter of the necessary antenna is roughly proportional to the wavelength. For instance, if we have a frequency of 500 Megahertz and thus a wavelength of about 60 centimeters, we would have an antenna with a diameter of at least 40 centimeters (this is a lower bound, since in reality $\eta_{\text {aper }}<1$ ). As long as we are able to accommodate a big enough antenna on our lander to meet our $\theta_{b}=90^{\circ}$ requirement, the lander antenna gain can be treated as independent of our frequency. Revisiting the Friis transmission equation, we now have an expression for our required RF input power in Eq. (15):

$$
P_{R F}=\frac{S 16 l^{2}}{6.828 d_{r}{ }^{2} \eta_{\text {med }} \eta_{\text {aper }, t} \eta_{\text {aper }, r} \eta_{\text {point }}}
$$

The wavelength-dependence cancels out. If we assume low absorption losses (a reasonable assertion if we operate with a wavelength smaller than 60 centimeters of, say, .3dB giving us an $\eta_{\text {medium }}$ of $\sim .933$, (and similarly a pointing error of $.3 \mathrm{~dB}$, i.e. $\eta_{\text {pointing }}=$ .93) and an $\eta_{\text {aper, } t}$ and $\eta_{\text {aper,r }}$ of .55, and use a 
$65^{\text {th }}$ International Astronautical Congress, Toronto, Canada. Copyright $@ 2014$ by the International Astronautical Federation. All rights reserved.

$d_{r}=\sqrt{2} \cdot 5$ meters (i.e. two antenna on the relay satellite having an aperture diameter of $5 \mathrm{~m}$, similar to the Boeing comm satellites, we get a power required in Eq. (16) of:

$$
P_{R F}=\frac{8.563 \cdot 10^{-17} \mathrm{Watts} \cdot 16 \cdot(55850 \mathrm{~km})^{2}}{6.828(\sqrt{2} \cdot 5 \mathrm{~m})^{2} .55 \cdot .55 \cdot .93 * .93}
$$

This is the minimum power required when the satellite is at the zenith. For the case of the satellite at the very edge of the $90^{\circ}$ beam where there is a $3 \mathrm{~dB}$ loss, the distance $l$ is less (in fact, it is about 28000 kilometers), leading to the observation that the signalto-noise ratio is in fact $3 \mathrm{~dB}$ better when on the edge of the $90^{\circ}$ beam because the relay satellite is much closer. Although it has not been proven for all points in between (since this depends on the exact shape of the antenna far-field pattern), one can expect that the power required at the zenith is greater than other places along the orbit in the $90^{\circ}$ beam or design the lander antenna to make it so, essentially designing the far-field pattern to match the orbit.

It should be mentioned at this point that traditionally a $3 \mathrm{~dB}$ (i.e. factor of $~ 2$ ) link margin is included in the above calculation, giving a total RF power required of almost 0.1 Watts. It is assumed the DC-to-RF power conversion efficiency is just $10 \%$ for the lander, which is in line with previous estimates but should be examined in more detail in future studies examining the state of the art of silicon carbide RF circuits. Thus, the final power required is $1 \mathrm{~W}$ att for the communications subsystem. 1 Watt is low enough that some cost-saving or performance-enhancing trades should be considered, for instance going with fewer and/or smaller receiving antenna on the relay satellite, considering a battery power supply instead of radioisotope (as is discussed elsewhere), allowing greater data availability, designing for a significantly lower word error rate, or allowing a higher data rate.

\section{IV.II.IV Orbital Relay}

A trade study was performed to determine which orbit the orbiter should be placed in. The chosen orbit depended on the location of the lander. Lander location choices were between a near equatorial site and a near polar site. In a near equatorial site for the lander, the orbit appears to move over the course of the mission. This means that the orbiter will slowly lose more sight of the lander over the mission duration. At the end of mission the orbiter will be out of beam of the lander. In a near polar site at 70 degrees latitude for the lander, the relay orbiter stays mostly in the beam pattern of the lander, which is desirable. The orbit and lander beam pattern for a near equatorial and near polar lander locations are compared in Fig. 8. The polar orbit and near polar lander site are chosen for the mission because the orbiter will spend more time in the beam pattern of the lander.

Communication from the orbiter to the Deep Space Network on Earth was assumed to be conventional, and was not analyzed in this study.

\section{IV.III Command and Data Handling (C\&DH)}

IV.III.I C\&DH Requirements and Assumptions

All electronics shall operate at Venus ambient temperature. The digitization scheme design shall be simple. It shall have forward error correction and detection. Chosen scheme shall be within lab-level technological capability at NASA Glenn Research Center. The landed mission will receive no commands from the ground. All operations will be preprogrammed.

Low-level Silicon Carbide (SiC) electronics integration is assumed. Number of gates will be in the range of hundreds. This assumption is based on $\mathrm{N}$ Channel MESFET SiC technology developed at Glenn Research Center. SiC has been demonstrated to operate at temperatures as high as $650^{\circ} \mathrm{C} .{ }^{35}$

A $\mathrm{SiC}$ transistor-based amplifier circuit has been demonstrated to operate at $500^{\circ} \mathrm{C} .{ }^{36}$ High-temperature low-level electronics will be used on the lander. 1.5 milliWatts per gate is assumed.

DC to RF conversion efficiency dominates power requirements for a given minimum data rate. The current assumption for this efficiency is about $10 \%$ for near-term $500^{\circ} \mathrm{C} \mathrm{SiC}$ amplifiers which operate in the Ultra High Frequency (UHF) regime. An Extended Hamming Code with 10 bits of data is used. A simple modulation baseline scheme chosen was binary phase shift key (BPSK) instead of the more crude binary frequency shift key (BFSK) which requires twice the signal-to-noise ratio to achieve the same error rate as BPSK.
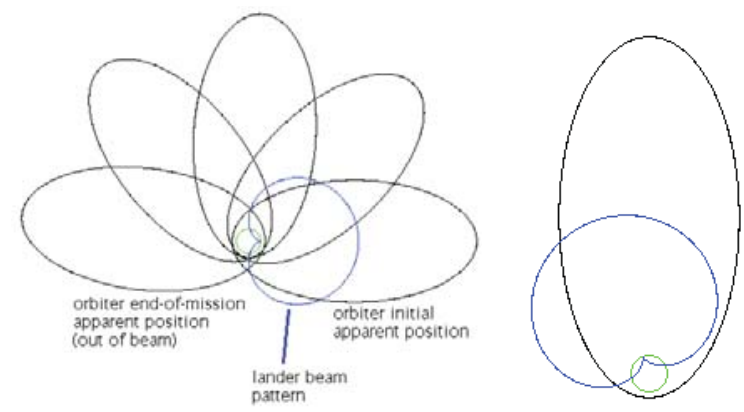

Fig. 8: Comparison of a communications for a nearequatorial landing site (left) and a near polar landing site (right). 




Fig. 9: Circuit with hamming code.

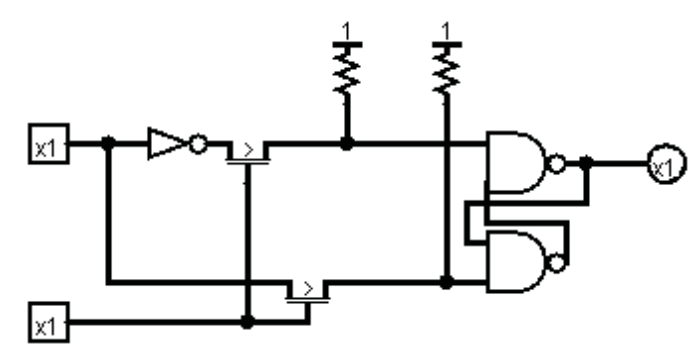

Fig. 10: Simplified latch for n-channel MESFETs.

\section{IV.III.II C\&DH Design and MEL}

The design for the Command and Data Handling subsystem involves 10 instrument channels, digitized to 10-bit precision, each sampled at about 25 Hertz. Some instruments are sampled doubled. There will be single error detecting extended Hamming code for forward error correction. Also the transmission will be continuous. Figs. 9 and 10 display some aspects for command and data handling. The first diagram shows the extended Hamming code in a circuit, while the second diagram shows the simplified latch for $\mathrm{n}$-channel MESFETs. ${ }^{37}$



Fig. 11: Spherical GPHS-RTG with dimensions.

\section{IV.III.III C\&DH Risk Inputs}

The lander will not be able to receive any commands from the orbiter. Therefore any failure will not have the capability of being fixed. Not all the data that the lander continuously uplinks will reach the orbiter. Therefore some information will be lost.

\section{IV.III.IV C\&DH Recommendation}

- Optimize RF communications.

- Decide on a placeholder antenna.

- Conventional stand-in circuit for simplified latch.

- Continue working on link budgets.

\section{IV.IV Electrical Power System for Lander}

The trade study for the power system of the lander compared a radioisotope power system (RPS) and primary sodium sulphur (NaS) batteries. The methodology and conclusions of this study are fully described in Salazar, et al. (2014). ${ }^{38}$ A customized General Purpose Heat Source - Radioisotope Thermal Generator (GPHS-RTG) is chosen for the RPS. It consists of 2 GPHS blocks, microtherm insulation, 64 Silicon Germanium (SiGe) thermoelectrics, a titanium pressure vessel wall, pyrolytic graphite fins, and a gas management valve. A proposed schematic can be seen in Figs. 11 and 12. The total electrical power output from this system is estimated to be 25.7 Watts with a total mass of $24 \mathrm{~kg}$.

\section{IV.V Electrical Power System for Descent Package \\ IV.V.I Power Requirements and Assumptions}

The descent package shall survive for about 5 hours during entry into the atmosphere of Venus. The power system for the descent package will be thermally insulated to allow the use of conventional electronics and batteries.

IV.V.II Power Design and MEL

As the descent package descends through the atmosphere, thermal mass will absorb heat leaking into the package. The components, electronics, instruments,

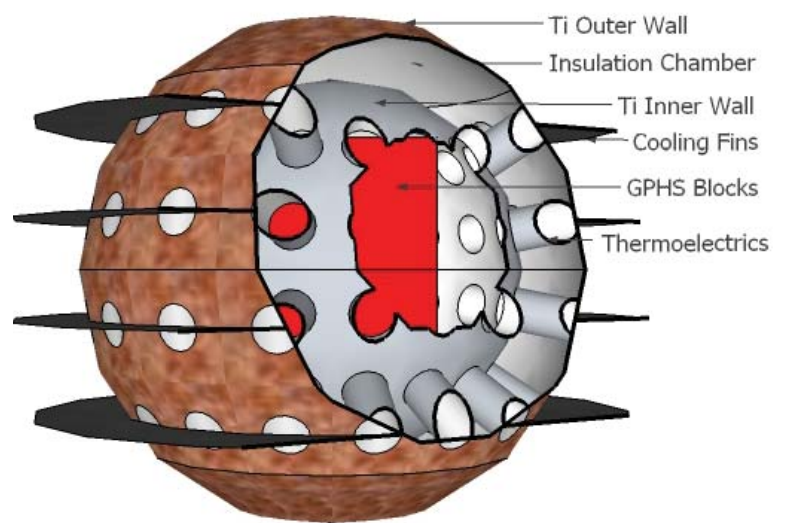

Fig. 12: Schematic cutaway of GPHS-RTG. 
and power supply within the package will survive until the thermal mass cannot absorb any more heat leaking in. Phase Change Material (PCM) will be the thermal mass. A titanium pressure vessel wall and insulation will surround the PCM and package. This vessel wall will be part of the aeroshell. The top of the aeroshell will house two parachutes for the package. At a specified altitude the lander will separate from the descent package. The batteries will begin to melt as the temperature inside the package rises.

The power system for the descent package will be composed of primary batteries. The descent package required 80 Watts as detailed in an earlier subsection. A $10 \%$ margin is added for safety. The power requirement for the descent package is therefore 88 Watts.

The Mars Exploration Rovers (MER) are using rechargeable Lithium-ion batteries for power assistance, the first space mission to use such batteries. ${ }^{39}$ Lithium ion batteries have many benefits which include mass, volume, energy efficiency, and self-discharge. ${ }^{39}$ Their wide operating range is between -20 to $40^{\circ} \mathrm{C}$ ( 253.15 to 303.15 Kelvin). ${ }^{39}$ That upper bound is much lower than the Venus ambient temperature. As the package descends, the PCM will absorb the heat leaking in, and the batteries will be able to operate for a short period of time. Once the operating temperature range is breached the batteries will not function well and will begin to melt. That is why it is critical to include enough insulation around the package to allow it to survive for the few hours needed. Lithium ion batteries are chosen for the short-lived descent package. To estimate the battery mass needed for the descent package, the following variables were used:

- An initial baseline power requirement of 140 Watts, assuming 90 Watts for the lander science package and $50 \mathrm{Watts}$ for the communications subsystem.

- A mission duration of 5 hours as it descends through the atmosphere.

- Charge efficiency of $100 \%$. $^{39}$

- Specific energy of 100 Watt hours per kilogram. ${ }^{39}$

An approximate battery capacity and mass were calculated to be 700 Watt hours and 7 kilograms respectively. The power management and distribution wires and components were assumed to be $10 \%$ of the total mass, therefore the final mass of the power system for the descent package is determined to be $9.2 \mathrm{~kg}$.

\section{IV.V.III Power Trades}

No trade study was done for the power system for the descent package.

\section{IV.V.IV Power Analytical Methods}

Simple calculations were performed to estimate the total mass for the power system of the descent package.

\section{IV.V.V Power Risk Inputs}

The batteries on the descent package are of less risk because they have already been built and tested. The Lithium ion batteries have been used on the twin Mars Exploration Rovers that were launched in 2003. ${ }^{39}$

\section{IV.V.VI Power Recommendation}

Thermal batteries are also compatible with the descent package because they can provide high-power for a short-duration. ${ }^{39}$ A trade study between Lithium ion batteries and thermal batteries could be performed.

\section{IV.VI Propulsion System}

Neither the lander nor the descent package will include a propulsion system. The lander is intended to be stationary for the duration of deployment. The descent package will utilize a parachute system during entry, passively impact the surface, then continue to function until the power system fails. The propulsion system for the orbiter is assumed to be conventional and it is not analyzed in this study.

\section{IV.VII Structures and Mechanisms System (SMS) \\ IV.VII.I SMS Requirements}

The lander must contain the necessary hardware for science payload, power, communications, and flight control. It must be able to withstand loads during launch, EDL, and surface deployment. All structures must withstand the high temperatures and pressures of the Venus environment, while resisting the corrosive atmosphere.

Mechanisms are required to function for single events and are used to separate from the LV, jettison the heat shield, jettison the lander, and to deploy the seismometer and antenna.

\section{IV.VII.II SMS Assumptions}

The lander is constructed primarily of a Ti alloy, Ti$6 \mathrm{Al}-4 \mathrm{~V}$, with the surfaces exposed to the atmosphere either coated with Titanium Oxide $\left(\mathrm{TiO}_{2}\right)$ or gold to inhibit corrosion by the high temperature atmosphere. Properties for the titanium alloy, Ti6Al4V, are from the Metallic Materials Properties Development and Standardization (MMPDS). ${ }^{40}$ Shape memory alloys will allow deployment of instruments without the use of motors. ${ }^{41}$ These materials will be thermally controlled with insulation during EDL and will only deploy the antenna and seismometer once they attain the ambient Venus temperature. ${ }^{42}$

The aeroshell and cruise deck are assumed to be of similar mass and design as that used in studies of previous Discovery class missions, such as the Advanced Lithium Ion Venus Explorer (ALIVE). ${ }^{4}$ This aeroshell was chosen to accommodate the expected growth of the HADES mission components and allow for additional landers to be deployed. 


\section{IV.VII.III SMS Design and MEL}

- The descent instrument suite is housed within a Ti enclosure in the aeroshell's backshell, surrounded by phase change salts composed of lithium nitrate trihydrate or a similar material to insulate the package. $^{43}$

- The main bus of the lander consists of a Ti sphere that houses the RTG and insulation. This provides the most efficient means of insulating the RPS blocks from the Venus environment, and saves weight by using the power supply as the primary structural element.

- Two packages are mounted at the top and bottom of the RTG sphere. On the top is a communications package that includes the shape memory alloydeployable helical antenna. Immediately beneath the sphere is the housing for three of the science instruments, the imager and the two atmospheric sensor suites.

- A larger Ti sphere is used to house the seismometer, which is mounted to the bottom of the science package by means of a shape memory alloy deployment mechanism. This sphere will be physically decoupled from the lander and placed in direct contact with the surface on a thin crushable Ti honeycomb footing.

- Landing gear is composed of rigid tubular Ti struts supporting an impact absorbing ring, made from a commercially available crushable Ti honeycomb. The landing gear is attached to the base of the science package.

- All exposed surfaces are anodized or plated with a layer of gold, or other suitable material, to resist the corrosive chemistry on Venus.

\section{IV.VII.IV SMS Trades}

No trades for structural design were considered for this study.

IV.VII.V SMS Analytical Methods

Analysis of the Ti alloy, Ti-6Al-4V, for use in pressure vessels on the surface of Venus may be performed using equation 17 from Young's and Budynas' Roark's Formulas for Stress and Strain (2002):

$$
P=\frac{0.365 E t^{2}}{r^{2}}
$$

The pressure differential across the spheres is approximately 9.20 MPa, and the Young's modulus of the material is $97 \mathrm{GPa}$. The RTG sphere has a radius of $12 \mathrm{~cm}$ and the seismometer has a $15 \mathrm{~cm}$ radius. Solving for the wall thickness, and applying a safety factor of
1.5 , results in a minimum thickness of approximately 3 $\mathrm{mm}$ for the RTG and $4 \mathrm{~mm}$ for the seismometer.

Final descent velocity can be calculated in Eq. (18).

$$
V_{\text {terminal }}=\left(\frac{2 m g}{\rho C_{D} A}\right)^{\frac{1}{2}}
$$

Where $V_{\text {terminal }}$ is the terminal velocity, $\mathrm{m}$ is the mass, $\mathrm{g}$ is the gravitational acceleration, $\rho$ is density, $C_{D}$ is the coeficiecnt of drag, and A is the surface area. The density is about 65 kilograms per meters squared. The lander has a total mass on the order of $100 \mathrm{~kg}$ and will have undergone parachute deceleration before landing. These factors are well within the heritage of previous mission designs that have used similar landing structures and should present no significant obstacle for future mission planners.

\section{IV.VII.VI SMS Risk Input}

As with all spacecraft, the loss of structural integrity due to vibration, g loads, or impact must be considered. In particular, the novel application of shape memory alloys in the mechanisms of the lander must be carefully constructed and insulated to prevent premature deployment.

Due to the mission's long duration in the corrosive Venus atmosphere, structural fatigue may reduce instrumentation performance or potentially cause failure in one or more of the support structures.

\section{IV.VII.VII SMS Recommendation}

The use of alternative materials, such as coated carbon fiber or ceramics, may allow mass savings, but should be analyzed for performance in the extreme environment of Venus. A detailed stress analysis is recommended to optimize the structural design and strength of the lander. Analysis of the final descent of the lander using computational fluid dynamics is recommended to ensure upright operations.

\section{IV.VIII Thermal Control}

\section{IV.VIII.I Lander Thermal Control}

Thermal control for the lander should be detailed for three modes of the mission: cruise, EDL, and science. During entry into the atmosphere the lander will be inside an aeroshell. The aeroshell will have an ablative heat shield. Once on the surface all of the components of the lander are assumed to operate at Venus ambient temperature and pressure. Thermal control for the lander involves channeling heat from the GPHS blocks through the thermoelectrics and then radiating it to the environment through the fins.

\section{IV.VIII.II Descent Package Thermal Control}

The descent package includes 22 kilograms of science instruments and 6 kilograms for its power 
system. The dish for its communications subsystem will operate at high temperatures and be attached to the package on the top. Therefore only the instruments and batteries will be insulated. Passive thermal management techniques will be used to minimize the heat leak into the descent package for its lifetime. Previous analyses have indicated that a 5-hour lifetime is achievable if insulation and phase change materials are employed for thermal control, and the use of passive thermal management techniques will make the descent package simpler and more robust. ${ }^{3}$ The Venus flagship mission study traded liquid-vapor Phase Change Material (PCM), solid-liquid PCM, and active refrigeration for a lander's thermal control system. They chose the solidto-liquid PCM for a 5-hour lifetime lander. The descent package is also intended to survive for about 5 hours. Therefore the same PCM will be utilized for the descent package for this mission. The chosen PCM is lithium nitrate trihydrate. The Venus Flagship Mission study noted "this material was successfully used by the Soviets on the Venera landers." 3 Because of this, an analysis of the heat leak into the package is not performed. It is assumed that the PCM will allow the descent package to function for about 5 hours. 50 kilograms of PCM and insulation for thermal control is assumed.

\section{VI.VIII.III Recommendations}

Thermal Control for the lander and descent package needs thermal analysis. The amount of time it takes to fall through the atmosphere and the exact temperature difference between the inside and outside of the package before entry should be used to find an accurate approximation of the mass of the PCM required.

\section{AREAS FOR FUTURE STUDY}

There are many areas for future analysis that have either been assumed from heritage and must be further specified or that were out of the scope of this preliminary study. The launch vehicle, transfer orbit, and cruise phase must all be developed, and the entry, descent and landing phase in particular must be fully detailed. This will include a determination of the precise timing of CONOPS events, a demonstration of the forces experienced by the lander and descent packages, an optimization of the lander's structural design for controlled free fall and landing, and an extensive thermal analysis of the descent package and shape memory mechanisms.

Based on the rationale of the SEIS experiment on InSight, HADES assumes that a single seismometer will provide enough data to answer the question of whether seismic activity exists on Venus, and will characterize the nature, frequency, and duration of such events. The placement of two additional landers would add redundancy to the mission and allow for the origin of seismic events to be triangulated, thereby providing significant improvement of the data. Further study is recommended to expand the scope of the mission to include these additional units.

\section{VI.I Cost}

\section{COST AND RISK}

The structure and proposed capabilities of the HADES mission are comparable in size and scope to previously completed Discovery Class missions, such as Mars Pathfinder. Most of the technologies employed are easily adapted from this rich heritage, and new systems are often reconfigurations of proven instruments using updated materials and components. It is therefor concluded that the HADES mission could be accomplished under a Discovery Class budget.

\section{VI.II Risk}

Much of the risk for the HADES mission lies with the low TRL technologies. The high temperature scientific instruments pose greater risk than the instruments with higher TRL, and the customized GPHS-RTG power system is a completely new design. There is no heritage mission that demonstrates the use of a GPHS-RTG on the surface of Venus; therefore the power system holds the greatest amount of risk, overall. Many of these risks can be safely managed, however, with the implementation of a rigorous testing regime that employs a simulated Venus environment, such as the Glenn Extreme Environment Rig.

\section{CONCLUSION}

An unmanned mission to the surface of Venus should be planned and executed. Such a mission would address fundamental questions about the formation and evolution of terrestrial planets, granting new insight into the history and future of Earth. By utilizing the latest developments in materials science and instrumentation, in combination with proven, heritage technologies, the exploration of Venus can be achieved quickly and inexpensively.

\section{ACKNOWLEDGMENTS}

The authors would like to thank Steven R. Oleson for his invaluable advice on mission architecture, Paul C. Schmitz for his guidance on radioisotope power systems, Gary W. Hunter for his assistance with the high temperature seismometer and other scientific instruments, Santo Padula for his advice on shape memory alloys, and Ralph Lorenz for his consultation on science goals. This work would not have been possible without the contributions from the NASA Glenn Space Academy and the Minnesota, Montana, and Texas Space Grant Consortia. 
$65^{\text {th }}$ International Astronautical Congress, Toronto, Canada. Copyright $@ 2014$ by the International Astronautical Federation. All rights reserved.

${ }^{1}$ Squyres, S. W., et al., 2011, "Vision and Voyages for Planetary Science in the Decade 2013-2022," 42nd Lunar and Planetary Science Conference, Houston, Texas.

2 NSSDC Chronology of Venus Exploration, NASA Goddard Space Center, http://nssdc.gsfc.nasa.gov/ imgcat/html/object_page/v13_vg261_262.html, accessed Aug. 13, 2014.

${ }^{3}$ Hall, J. L., et al., 2009, "Venus Flagship Mission Study," NASA, Jet Propulsion Laboratory, California Institute of Technology, Task Order NM0710851.

${ }^{4}$ Oleson, S. R., "COMPASS Final Report: Advanced Lithium Ion Venus Explorer (ALIVE)," NASA, Glenn Research Center, CD-2012-72.

${ }^{5}$ Hoffman, J. H., et al., 1979, "Composition and Structure of the Venus Atmosphere: Results from Pioneer Venus," Science, Vol. 205, No. 4401, pp. 49-52.

${ }^{6}$ Beatty, J. K., et al., 1999, The New Solar System, $4^{\text {th }}$ ed., Sky Publishing Corporation and the Press Syndicate of the University of Cambridge, Massachusetts, Chap. 8.

${ }^{7}$ Colozza, A. J., 2012, "Radioisotope Stirling Engine Powered Airship for Low Altitude Operation on Venus." NASA/CR-2012-217665.

${ }^{8}$ Solar System Exploration, NASA, https://solarsystem.nasa.gov/planets/, accessed Aug. 14, 2014.

${ }^{9}$ Baker, C., et al., 2010, Venus Intrepid Tessera Lander (VITaL)," NASA, Goddard Space Flight Center.

10 Schofield, J. T., et al., 1997, "The Mars Pathfinder Atmospheric Structure Investigation/Meteorology (ASI/MET) Experiment,” Science, Vol. 278, No. 5344, pp. 1752-1758.

${ }^{11}$ Waite, J. H., et al., 2004, “The Cassini Ion and Neutral Mass Spectrometer (INMS) Investigation," Space Science Reviews, Vol. 114, pp. 113-231.

${ }^{12}$ Mahaffy, P. R., et al., 2012, “The Sample Analysis at Mars Investigation and Instrument Suite," Space Science Reviews, Vol. 170, No. 1-4, pp. 401-478.

${ }^{13}$ Sromovsky, L. A., et al., 1980, "Pioneer Venus Small Probes Net Flux Radiometer Experiment," Geoscience and Remote Sensing, IEEE Transactions on, Vol. GE-18, No. 1, pp. 117-122.

${ }^{14}$ Sromovsky, L. A., et al., 1992, "Galileo Net Flux Radiometer Experiment," Space Science Reviews, Vol. 60, pp. 233-262.

${ }^{15}$ Russell, C.T., et al., 1980, "Pioneer Venus Orbiter Fluxgate Magnetometer," Geoscience and Remote Sensing, IEEE Transactions on, Vol. GE-18, No. 1, pp. 32-35.

${ }^{16}$ Dougherty, M. K., et al, 2005, "Cassini Magnetometer Observations During Saturn Orbit Insertion," Science, Vol. 307, No. 5713, pp. 1266-1270.

${ }^{17}$ Ragent, B., et al, 1980, "Pioneer Venus Sounder and Small Probes Nephelometer Instrument," Geoscience and Remote Sensing, IEEE Transactions on, Vol. GE-18, No. 1, pp. 111-117.

18 Takahashi, Y., et al., 2008, "Lightning Detection by LAC Onboard the Japanese Venus Climate Orbiter, Planet-C," Space Science Review, Vol. 137, pp. 317-334.

${ }^{19}$ Scarf, F. L., et al., 1980, "Lightning on Venus - Orbiter Detection of Whistler Signals," Journal of Geophysical Research, Vol. 85, pp. 8158-8166.

${ }^{20}$ Malin, M. C., et al, 2005, "The Mast Cameras and Mars Descent Imager (MARDI) for the 2009 Mars Science Laboratory," 36th Annual Lunar and Planetary Science Conf., Vol. 36, League City, Texas, pp. 1214.

21 Glenn Extreme Environment Rig (GEER), NASA Glenn Research Center, http:// spaceflightsystems.grc.nasa.gov/SSPO/SS/Extreme/, accessed Aug. 31, 2014.

${ }^{22}$ Robert, O., et al., 2012, “A Martian Very Broad Band (VBB) Seismometer," International Workshop on Instrumentation for Planetary Missions, IPM-2012.

${ }^{23}$ Hunter, G. W., et al., 2012, "Development of a High Temperature Venus Seismometer and Extreme Environment Testing Chamber," International Workshop on Instrumentation for Planetary Missions, IPM-2012.

${ }^{24}$ Stauffer, J.-M., 2006, "Current Capabilities of MEMS Capacitive Accelerometers in a Harsh Environment," Aerospace and Electronic Systems Magazine, IEEE, Vol. 21, No. 11, pp. 29-32.

${ }^{25}$ Interior Exploration using Seismic Investigations, Geodesy and Heat Transport, Centre National d'Etudes Spatiales (CNES), http://smsc.cnes.fr/INSIGHT/GP_seis.htm, accessed Aug. 31, 2014.

${ }^{26}$ Asakawa, K., et al., 2012, "Ceramic Pressure-Tight Housings for Ocean-Bottom Seismometers Applicable to 11-km Water Depth," Oceanic Engineering, IEEE Journal of, Vol. 37, No. 4, pp. 756-763.

${ }^{27}$ Huck, F., et al., 1975, “The Viking Mars Lander Camera,” Space Science Instrumentation, Vol. 1, pp. $189-241$.

${ }^{28}$ Landis, G. A. and Haag, E., 2013, "Solar Cell Performance on Venus," AIAA $11^{\text {th }}$ International Energy Conversion Engineering Conference, San Jose, California.

${ }^{29}$ Camera, Imager, Viking Mars Lander, Smithsonian National Air and Space Museum, http://airandspace.si.edu/ collections/artifact.cfm?object=nasm_A19810661000\#myCarousel, accessed Aug. 31, 2014. 
${ }^{30}$ Gas Sensor Array, www.makelengineering.com, accessed Aug. 1, 2013.

${ }^{31} \mathrm{Ji}$, J., et al., 2008, "High Temperature Mechanisms for Venus Exploration," 37th COSPAR Scientific Assembly, Vol. 1, Montreal, Canada, pp. 1370.

32 Hunter, G. W., et al., 1998, "A Hazardous Gas Detection System for Aerospace and Commercial Applications," American Institute of Aeronautics and Astronautics, NASA, Cleveland, Ohio.

${ }^{33}$ Ferri, F., et al., 2002, "Huygens Atmospheric Structure Instrument of Huygens Probe on Cassini Mission," Acta Astronautica, Vol. 30, No. 4, pp. 249-255.

${ }^{34}$ Anderson, H.R., 2003, Fixed Broadband Wireless System Design, John Wiley \& Sons, USA, pp. 206-207.

${ }^{35}$ Silicon Carbide Electronics and Sensors, http://www.grc.nasa.gov/WWW/SiC/, accessed Aug. 31, 2014.

${ }^{36}$ Electronics, http://www.grc.nasa.gov/WWW/RT/2006/RI/RIS-neudeck.html, accessed Aug. 31, 2014.

${ }^{37}$ Wireless Communications: Principles and Practice, Rappaport $2^{\text {nd }}$ edition

${ }^{38}$ Salazar, et al., 2014, "Non-Cooled Power System for Venus Lander," American Institute of Aeronautics and Astronautics, AIAA-2014-3459, Cleveland, Ohio.

${ }^{39}$ Bugga, R., et al., 2006, "Li-ion Rechargeable Batteries on Mars Exploration Rovers," NASA, Jet Propulsion Laboratory, California Institute of Technology, Pasadena, CA.

${ }^{40}$ MMPDS Ti-6Al-4V, Metallic Materials Properties Development and Standardization (MMPDS), MMPDS-02 Vol. 4, Federal Aviation Administration, 2006.

${ }^{41}$ Noebe, R., et al., 2006, "Properties of a Ni19.5Pd30Ti50.5 high-temperature shape memory alloy in tension and compression," Smart Structures and Materials 2006: Active Materials: Behavior and Mechanics, Proc. SPIE 6170 .

${ }^{42}$ Stebner, A., et al., 2009, "Development, Characterization, and Design Considerations of $\mathrm{Ni}_{19.5} \mathrm{Ti}_{50.5} \mathrm{Pd}_{25} \mathrm{Pt}_{5}$ High-temperature Shape Memory Alloy helical Actuators," Journal of Intelligent Material Systems ads Structures, Vol. 20, pp. 2107-2126.

${ }^{43}$ Pauken, M., et al., 2010, "Thermal Control Technology Developments for a Venus Lander”, AIP Conference Proceedings, Vol. 1208, pp. 68. 